\title{
Qualidade de vida de estudantes de mestrado e doutorado em enfermagem
}

\author{
Maria José Quina Galdino ${ }^{1}$, Júlia Trevisan Martins², Renata Perfeito Ribeiro ${ }^{3}$, Alessandro Rolim Scholze 4 \\ Sirlene Aparecida Scarpin Tsukamoto ${ }^{5}$, Maria do Carmo Fernandez Lourenço Haddad ${ }^{6}$
}

\section{RESUMO}

Este estudo objetivou analisar a qualidade de vida e seus preditores entre estudantes de mestrado e doutorado em enfermagem. Pesquisa transversal com 129 pós-graduandos de três universidades públicas da Região Sul do Brasil. Para coleta utilizou-se um questionário para caracterização e o WHOQOL-Bref. Os dados foram analisados por estatística descritiva e múltipla. Qualidade de vida física obteve o maior valor mediano e as relações sociais, o menor. Sedentarismo, insatisfação com o tema de pesquisa, dificuldade de conciliar estudos e vida pessoal, tabagismo, consumo de bebidas alcoólicas, preocupação com o prazo para conclusão do curso e relacionamento insatisfatório com os docentes estiveram associados significativamente às menores percepções de qualidade de vida. Houve indícios que a formação stricto sensu impactou negativamente a qualidade de vida dos pesquisados, visto que a maioria dos fatores associados que a influenciaram desfavoravelmente foram variáveis relacionadas ao contexto acadêmico.

Descritores: Qualidade de Vida; Educação de Pós-graduação em Enfermagem; Estudantes de Enfermagem.

\footnotetext{
${ }^{1}$ Enfermeira, Mestre em Enfermagem. Discente do Programa de Pós-Graduação em Enfermagem, nível Doutorado, da Universidade Estadual de Maringá. Professora Assistente da Universidade Estadual do Norte do Paraná. Bandeirantes, PR, Brasil. E-mail: mariagaldino@uenp.edu.br.

2 Enfermeira, Doutora em Enfermagem. Professora Adjunta da Universidade Estadual de Londrina. Londrina, PR, Brasil. E-mail: jtmartins@uel.br.

${ }^{3}$ Enfermeira, Doutora em Enfermagem. Professora Adjunta da Universidade Estadual de Londrina. Londrina, PR, Brasil. E-mail: perfeitorenata@gmail.com.

${ }^{4}$ Enfermeiro, Mestre em Enfermagem, Professor da Universidade Estadual do Norte do Paraná. Bandeirantes, PR, Brasil. E-mail: scholze@uenp.edu.br.

${ }^{5}$ Enfermeira. Discente do Programa de Pós-Graduação em Enfermagem, nível Mestrado, da Universidade Estadual de Londrina. Londrina, PR, Brasil. E-mail: sirlene.tsukamoto@hotmail.com.

${ }^{6}$ Enfermeira, Doutora em Enfermagem Fundamental. Professora Associada do Departamento de Enfermagem da Universidade Estadual de Londrina. Londrina, PR, Brasil. E-mail: carmohaddad@gmail.com.
}
Artigo recebido: 14/12/2017.
Artigo aprovado: 09/05/2018.
Artigo publicado: 31/12/2018.

\section{Como citar esse artigo:}

Galdino MJQ, Martins JT, Ribeiro RP, Scholze AR, Tsukamoto SAS, Haddad MCFL. Qualidade de vida de estudantes de mestrado e doutorado em enfermagem. Rev. Eletr. Enf. [Internet]. 2018 [acesso em:

];20:v20a41. Disponível em: https://doi.org/10.5216/ree.v20.50673. 


\section{INTRODUÇÃO}

A pós-graduação stricto sensu em enfermagem iniciou-se nos Estados Unidos da América (EUA) na década de 1930, com a criação dos primeiros mestrados e doutorados. Ao longo dessas oito décadas, a expansão deste nível de ensino para os enfermeiros tem sido um fenômeno mundial. No Brasil, surgiu em 1972, com a criação do curso de mestrado na Escola de Enfermagem Anna Nery, da Universidade Federal do Rio de Janeiro. Em 1981, teve início o primeiro curso de doutorado: o Programa Interunidades de Doutoramento em Enfermagem, parceria da Escola de Enfermagem com a Escola de Enfermagem de Ribeirão Preto, ambas da Universidade de São Paulo ${ }^{(1-3)}$.

No início de 2018, havia no Brasil 76 Programas de Pós-Graduação vinculados à área da Enfermagem (PPGEnf), que ofertavam 112 cursos, dos quais 51 eram mestrados acadêmicos, 38 doutorados e 23 mestrados profissionais ${ }^{(4)}$. Esses programas têm como finalidade aprofundar o conhecimento da área e qualificar enfermeiros para o ensino, pesquisa e assistência de enfermagem ${ }^{(3)}$.

Com tais objetivos a alcançar, mestrandos e doutorandos são inseridos em diversas atividades, como: cursar créditos relativos a disciplinas teóricas; ministrar aulas para outros níveis de formação; participar do grupo de pesquisa e das pesquisas de seu orientador, bem como participar da orientação de alunos de iniciação científica e trabalho de conclusão de curso; divulgar resultados de pesquisa em forma de artigos e apresentações em eventos científicos; elaborar sua dissertação/tese, entre outras. Assim, a formação stricto sensu, pelas numerosas atividades exigidas do estudante, muitas vezes, em concomitância com trabalho e afazeres domésticos, pode sobrecarregá-lo e provocar uma repercussão negativa no bem-estar e na saúde física e mental e, por consequência, interferir em sua qualidade de vida $(Q V)^{(5-6)}$.

A definição de QV é ampla, multidimensional e deve ser interpretada de acordo com a percepção subjetiva de cada pessoa, acerca da satisfação com vários aspectos de sua vida, entre eles a condição física, psicológica, relacionamentos sociais e ambiente onde vive ${ }^{(7)}$. Diante desta abrangência, há diversos conceitos para esta expressão. Todavia, neste estudo adotou-se a concepção proposta pela Organização Mundial da Saúde (OMS) que conceitua QV como "a percepção do indivíduo de sua posição na vida no contexto da cultura e sistema de valores nos quais ele vive e em relação a seus objetivos, expectativas, padrões e preocupações"(8).

Estudos sobre a QV das pessoas tem despertado o interesse dos pesquisadores, em vertentes relacionadas às condições clínicas, classes ocupacionais e no processo de formação profissional, pelo fato de ser temporal e influenciada pelas circunstâncias vividas. No âmbito de formação profissional na área de enfermagem identificouse apenas investigações com graduandos ${ }^{(9-11)}$. Nesse contexto, a QV foi associada à satisfação das necessidades humanas básicas (saúde, lazer, descanso) e de expectativas individuais, ao prazer e bem-estar, como também à experiência vivida na universidade ${ }^{(10)}$. Além disso, a satisfação pessoal durante o processo de formação parece estar estreitamente relacionada com a percepção de $Q V^{(11)}$.

Assim, investigar a QV de mestrandos e doutorandos em enfermagem poderá fornecer informações sobre suas perspectivas de bem-estar, necessidades, dificuldades, entre outros fatores importantes que são pouco conhecidos pela escassez de estudos com estes indivíduos ${ }^{(5)}$. O conhecimento gerado a partir deste estudo poderá subsidiar o desenvolvimento de estratégias que promovam a QV desses pós-graduandos, a fim de produzir um ambiente favorável ao aprendizado e à formação acadêmica de pesquisadores. 
Diante dessas considerações, objetivou-se, com este estudo, analisar a qualidade de vida e seus preditores entre estudantes de mestrado e doutorado em enfermagem.

\section{MÉTODO}

A pós-graduação stricto sensu em enfermagem iniciou-se nos Estados Unidos da América (EUA) na década de 1930, com a criação dos primeiros mestrados e doutorados. Ao longo dessas oito décadas, a expansão deste nível de ensino para os enfermeiros tem sido um fenômeno mundial. No Brasil, surgiu em 1972, com a criação do curso de mestrado na Escola de Enfermagem Anna Nery, da Universidade Federal do Rio de Janeiro. Em 1981, teve início o primeiro curso de doutorado: o Programa Interunidades de Doutoramento em Enfermagem, parceria da Escola de Enfermagem com a Escola de Enfermagem de Ribeirão Preto, ambas da Universidade de São Paulo(13).

No início de 2018, havia no Brasil 76 Programas de Pós-Graduação vinculados à área da Enfermagem (PPGEnf), que ofertavam 112 cursos, dos quais 51 eram mestrados acadêmicos, 38 doutorados e 23 mestrados profissionais $^{(4)}$. Esses programas têm como finalidade aprofundar o conhecimento da área e qualificar enfermeiros para o ensino, pesquisa e assistência de enfermagem ${ }^{(3)}$.

Com tais objetivos a alcançar, mestrandos e doutorandos são inseridos em diversas atividades, como: cursar créditos relativos a disciplinas teóricas; ministrar aulas para outros níveis de formação; participar do grupo de pesquisa e das pesquisas de seu orientador, bem como participar da orientação de alunos de iniciação científica e trabalho de conclusão de curso; divulgar resultados de pesquisa em forma de artigos e apresentações em eventos científicos; elaborar sua dissertação/tese, entre outras. Assim, a formação stricto sensu, pelas numerosas atividades exigidas do estudante, muitas vezes, em concomitância com trabalho e afazeres domésticos, pode sobrecarregá-lo e provocar uma repercussão negativa no bem-estar e na saúde física e mental e, por consequência, interferir em sua qualidade de vida $(Q V)^{(5-6)}$.

A definição de QV é ampla, multidimensional e deve ser interpretada de acordo com a percepção subjetiva de cada pessoa, acerca da satisfação com vários aspectos de sua vida, entre eles a condição física, psicológica, relacionamentos sociais e ambiente onde vive ${ }^{(7)}$. Diante desta abrangência, há diversos conceitos para esta expressão. Todavia, neste estudo adotou-se a concepção proposta pela Organização Mundial da Saúde (OMS) que conceitua QV como “a percepção do indivíduo de sua posição na vida no contexto da cultura e sistema de valores nos quais ele vive e em relação a seus objetivos, expectativas, padrões e preocupações”(8).

Estudos sobre a QV das pessoas tem despertado o interesse dos pesquisadores, em vertentes relacionadas às condições clínicas, classes ocupacionais e no processo de formação profissional, pelo fato de ser temporal e influenciada pelas circunstâncias vividas. No âmbito de formação profissional na área de enfermagem identificouse apenas investigações com graduandos ${ }^{(9-11)}$. Nesse contexto, a QV foi associada à satisfação das necessidades humanas básicas (saúde, lazer, descanso) e de expectativas individuais, ao prazer e bem-estar, como também à experiência vivida na universidade ${ }^{(10)}$. Além disso, a satisfação pessoal durante o processo de formação parece estar estreitamente relacionada com a percepção de $Q V^{(11)}$.

Assim, investigar a QV de mestrandos e doutorandos em enfermagem poderá fornecer informações sobre suas perspectivas de bem-estar, necessidades, dificuldades, entre outros fatores importantes que são pouco 
conhecidos pela escassez de estudos com estes indivíduos ${ }^{(5)}$. O conhecimento gerado a partir deste estudo poderá subsidiar o desenvolvimento de estratégias que promovam a QV desses pós-graduandos, a fim de produzir um ambiente favorável ao aprendizado e à formação acadêmica de pesquisadores.

Diante dessas considerações, objetivou-se, com este estudo, analisar a qualidade de vida e seus preditores entre estudantes de mestrado e doutorado em enfermagem.

\section{RESULTADOS}

Dos 129 participantes de pesquisa, 116 (89,9\%) eram do sexo feminino, $76(58,9 \%)$ com relacionamento conjugal estável e 81 (62,8\%) sem filhos. A idade mediana foi de 31 anos, variando entre 22 e 61 anos. A renda familiar mensal variou entre $\mathrm{R} \$ 1.500,00$ e $\mathrm{R} \$ 40.000,00$, com mediana de $\mathrm{R} \$ 7.600,00$ (US\$526,00, US\$14.035,00 e US\$2.667,00, respectivamente, em fevereiro 2015).

Com relação às características acadêmicas, 79 (61,2\%) eram mestrandos e 50 (38,8\%) doutorandos, que destinavam para o estudo até 15 horas diárias, com mediana de quatro horas. Sobre a satisfação com o tema de pesquisa, em escala de zero a 10, $113(87,6 \%)$ dos pós-graduandos referiram pontuação igual ou superior a sete pontos. Quanto a dedicação à pós-graduação, $46(35,7 \%)$ dedicavam-se exclusivamente ao curso e os demais conciliavam estudo e trabalho, dos quais $48(37,2 \%)$ atuavam na assistência de enfermagem e 35 (27,1\%) eram docentes de graduação ou cursos técnicos.

No que concerne aos hábitos de vida, 62 (48,1\%) dos investigados praticavam atividade física regularmente, com frequência de uma a três vezes por semana. Com relação ao uso de álcool, 50 (38,8\%) referiram consumir bebidas alcoólicas ao menos quatro dias no mês, 18 (14\%) afirmaram beber semanalmente e dois $(1,6 \%)$ relataram consumir diariamente. No que tange ao tabagismo, sete $(5,4 \%)$ eram fumantes ativos, dos quais, três (2,4\%), fumavam mais de 10 cigarros diariamente.

Na Tabela 1 verificou-se, entre os domínios de QV, que a menor mediana obtida pelos participantes foi no domínio relações sociais.

Tabela 1: Medidas descritivas de qualidade de vida dos estudantes de mestrado e doutorado em enfermagem. Região Sul, Brasil, 2015.

\begin{tabular}{cccc}
\hline WHOQOL-Bref & Mediana & Amplitude interquartílica (p25-p75) & Mínimo-Máximo \\
\hline QV geral & 90,0 & $82,5-98,0$ & $46-100$ \\
QV física & 67,9 & $57,1-75,0$ & $25-96$ \\
QV psicológica & 62,5 & $54,2-70,8$ & $25-92$ \\
QV relações sociais & 58,3 & $50,0-75,0$ & $17-100$ \\
QV meio ambiente & 59,4 & $53,1-68,7$ & $25-94$ \\
\hline
\end{tabular}

Quanto aos itens que compõem os domínios, as menores medianas foram para "sono e repouso" (domínio físico), "sentimentos positivos" (domínio psicológico), “atividade sexual” (domínio relações sociais) e "participação em oportunidades de recreação ou lazer" (domínio meio ambiente).

A Tabela 2 apresenta o modelo de análise de regressão linear múltipla para a QV geral. O modelo mostrouse estatisticamente significativo $(p<0,001)$, apresentando coeficiente de correlação múltipla de $R=0,600$ e de determinação $R^{2}=0,360$. Evidenciou-se que as variáveis dificuldade de conciliar estudos e vida pessoal, prazo para conclusão do curso, tabagismo e consumo de bebidas alcoólicas estiveram inversamente associadas à QV geral, 
isto é, predizem menor percepção de QV. Em contrapartida, quanto melhor o relacionamento com os docentes do curso, a satisfação com o tema de pesquisa e realizar exercícios físicos, maior foi a percepção de QV.

Tabela 2: Fatores preditores da qualidade de vida dos estudantes de mestrado e doutorado em enfermagem. Região Sul, Brasil, 2015.

\begin{tabular}{ccc}
\hline Modelo & Beta & Valor de $\boldsymbol{p}$ \\
\hline Atividade física & 3,365 & 0,058 \\
Satisfação com o tema de pesquisa & 1,142 & 0,031 \\
Dificuldade de conciliar estudos e vida pessoal & $-4,832$ & 0,009 \\
Tabagismo & $-12,243$ & 0,003 \\
Consumo de bebidas alcoólica & $-4,641$ & 0,013 \\
Preocupação com o prazo para conclusão do curso & $-9,164$ & $<0,001$ \\
Relacionamento com os docentes & 2,477 & 0,074 \\
\hline
\end{tabular}

\section{DISCUSSÃO}

A caracterização sociodemográfica revelou que prevaleceram mulheres jovens, com relacionamento conjugal estável e sem filhos. Isso indica que os enfermeiros têm optado pelo ingresso na pós-graduação stricto sensu logo após concluir seus estudos de graduação, como também têm postergado a constituição de família com filhos, provavelmente pela dedicação de tempo que este nível de ensino exige. Além disso, para a mulher contemporânea, a carreira profissional está tão atrelada à sua realização pessoal quanto a maternidade(12-13).

Verificou-se que a maioria dos estudantes se dedicava parcialmente à pós-graduação por possuir um vínculo empregatício, o que pode ter ocorrido pelo número reduzido de bolsas de estudo concedidas aos programas pelas agências de fomento. Embora no Plano Nacional de Pós-Graduação haja uma estimativa de, até 2020, duplicar a concessão do número de bolsas de mestrado e triplicar as de doutorado, os valores de bolsas praticados no ano de $2015-\mathrm{R} \$ 1.500,00$ (US\$526,00) para o mestrado e $\mathrm{R} \$ 2.200,00$ (US\$772,00) para o doutorado - não são suficientes para o provimento desses pós-graduandos, que acabam por depender de financiamento familiar ${ }^{(14-17)}$.

Os diversos papéis desempenhados por esses pós-graduandos relacionados ao estudo, trabalho e família podem estar vinculados às menores médias de satisfação com sono e repouso, energia e fadiga, atividade sexual, participação em oportunidades de lazer e sentimentos positivos (aproveitar a vida). Em estudo realizado nos EUA sobre o bem-estar de 194 doutorandos, foi demonstrado que eles diminuem o tempo destinado a repouso e exercícios físicos para conseguir atender às demandas da pós-graduação(18). Supõe-se, desse modo, que a atividade sexual e o lazer também tenham sido deixados em segundo plano em virtude da escassez de tempo.

Os mestrandos e doutorandos apresentaram percepções inferiores de QV quando comparados aos estudantes de graduação e a outros grupos ocupacionais de enfermeiros ${ }^{(11,19-20)}$, sugerindo que este nível de ensino possui demandas que interferem desfavoravelmente na QV dessas pessoas.

Pressupõe-se que estes estudantes suportam o impacto sofrido em sua QV porque acreditam que o intenso ritmo de vida tem prazo para terminar. Além disso, como no trabalho, o estudo é permeado por vivências de prazer e satisfação, que podem motivar os indivíduos a continuar ${ }^{(9,21)}$, tais como: o privilégio de estar cursando este nível de ensino, o reconhecimento de seu talento científico, o crescimento profissional e melhores oportunidades de ganhos financeiros ${ }^{(22)}$. 
O prazo para a conclusão do curso e a dificuldade de conciliar a vida de pós-graduando com a vida pessoal já foram indicados, em outros estudos ${ }^{(15-16)}$, como preocupações comuns entre mestrandos e doutorandos, e neste estudo, associaram-se inversamente à QV. Dessa forma, administrar o tempo frente às diversas atividades desse indivíduo é um desafio. A organização do tempo pode ser determinante no ajustamento a esse processo e também no seu bem-estar, por permitir o equilíbrio entre estudos e vida pessoal, não sendo necessário abdicar de atividades prazerosas como lazer, estar na companhia de familiares e amigos, tão essenciais quanto o título de mestre ou doutor.

Verificou-se que o sedentarismo, o tabagismo e o consumo de bebidas alcoólicas foram preditores de menor percepção da QV. Pesquisa desenvolvida em Nova York com 2.508 pós-graduandos da área de ciências da saúde demonstrou que os mestrandos e doutorandos que praticavam atividade física regularmente apresentaram melhor QV e saúde física e mental ${ }^{(23)}$. Este estudo também indicou que o álcool e o tabaco são as substâncias psicoativas mais usadas por estes estudantes para aliviar as tensões e o estresse ${ }^{(23)}$. Entretanto esses hábitos causam prejuízo à vida acadêmica por se relacionarem à dificuldades de aprendizagem ${ }^{(6)}$. Além disso, o uso de substâncias psicoativas pelos indivíduos pode estar relacionado a um mecanismo compensatório para resistir as pressões psíquicas, como uma tentativa de conjurar o adoecimento e o sofrimento ${ }^{(21)}$.

O relacionamento interpessoal com os membros do corpo docente do curso gerou impacto positivo na QV dos mestrandos e doutorandos desta pesquisa. Essa relação satisfatória é compreendida, pela maioria dos estudantes, como um dos aspectos mais relevantes em sua experiência acadêmica, que favorece a QV dentro da universidade, tanto no nível de formação stricto sensu, como na graduação. Ademais, os docentes podem oferecer aos estudantes a oportunidade de adquirir novas informações e habilidades, e apoio intelectual e emocional para enfrentar suas dificuldades acadêmicas ${ }^{(10,24-25)}$.

A satisfação com o tema de pesquisa também se associou favoravelmente à QV, ou seja, quanto mais satisfeito com esse quesito, melhor era a percepção de QV. O tema de pesquisa é o objeto principal da dissertação/tese, que exigirá mais envolvimento do pós-graduando, pois possui um nível de complexidade maior, quando comparado às outras pesquisas já realizadas pelo estudante ${ }^{(5)}$.

Dessa forma, este estudo indica que pesquisar um assunto que se considera relevante, com o qual se teve afinidade, foi um favorecedor de QV entre os estudantes de mestrado e doutorado. Assim, orientadores e orientandos devem dialogar sobre o tema de pesquisa e a trajetória a ser percorrida na investigação, devendo o estudante explicitar suas afinidades, anseios e dificuldades, o que favorece o estabelecimento de um consenso, além de promover um ambiente favorável à formação acadêmica. Recomenda-se, também, àqueles que possuem interesse neste nível de formação que estabeleçam contato prévio ao ingresso no curso, com um provável orientador que atue em linhas de pesquisa afins.

Embora os objetivos deste estudo tenham sido alcançados, houve limitações relacionadas ao delineamento transversal, que não permite inferências de causalidade. Os dados coletados foram autorrelatados, situação em que pode ocorrer influência dos padrões socialmente aceitáveis na reposta ao instrumento. Além disso, a amostra foi restrita a estudantes de mestrado e doutorado em enfermagem de três PPGEnf de universidades públicas da Região Sul do Brasil, o que, em razão da especificidade, limita a generalização dos seus achados. Apesar desses 
limites, os resultados obtidos neste estudo poderão subsidiar a elaboração e execução de políticas e programas de promoção do bem-estar e da QV no âmbito universitário.

Recomenda-se a sua replicação em outras regiões do país e, ainda, a realização de investigações com os docentes desses programas, pois há probabilidade de vivenciarem situações semelhantes que podem ser prejudiciais à sua QV.

\section{CONCLUSÃO}

Houve indícios de que a formação stricto sensu impactou negativamente a QV, visto que sua percepção desfavorável entre os mestrandos e doutorandos pesquisados foi associada, sobretudo, com aspectos do cotidiano acadêmico. Quanto aos preditores, revelou-se que realizar atividade física, o bom relacionamento com os docentes do curso e a satisfação com o tema de pesquisa favorecem a QV, enquanto dificuldade de conciliar estudos e vida pessoal, prazo para conclusão do curso, tabagismo e consumo de bebidas alcoólicas foram desfavoráveis à QV.

\section{REFERÊNCIAS}

1. Ketefian S, Redman RW. A critical examination of developments in nursing doctoral education in the United States. Rev Lat Am Enfermagem [Internet]. 2015 [cited 2018 Dec 31];23(3):363-71. Available from: https://doi.org/10.1590/0104-1169.0797.2566. 2. Morin KA. Nursing education: The past, present and future. J Heal Spec [Internet]. 2014 [cited 2018 Dec 31];2(4):135-41. Available from: https://doi.org/10.4103/1658-600X.142781.

3. Scochi CGS, Munari DB, Gelbcke FL, Erdmann AL, Gutiérrez MGR, Rodrigues RAP. Pós-graduação Stricto Sensu em Enfermagem no Brasil: avanços e perspectivas. Rev Bras Enferm [Internet]. 2013 Sep;66(spe):80-9. Available from: https://doi.org/10.1590/S0034-71672013000700011.

4. Coordenação de Aperfeiçoamento de Pessoal de Nível Superior. Plataforma Sucupira. Cursos Avaliados e Reconhecidos. Área de Avaliação. Área de Conhecimento. [Internet]. Brasil (BR): Coordenação de Aperfeiçoamento de Pessoal de Nível Superior; c2016 [cited 2018 Dec 31]. Available from:

https://sucupira.capes.gov.br/sucupira/public/consultas/coleta/programa/quantitativos/quantitativoAreaConhecimento.jsf?areaA valiacao=20.

5. Faro A. Um modelo explicativo para o bem-estar subjetivo: estudo com mestrandos e doutorandos no Brasil. Psicol. Reflex. Crit. [Internet]. 2013;26(4):654-62. Available from: https://doi.org/10.1590/\$0102-79722013000400005.

6. Kernan W, Bogart J, Wheat ME. Health-related barriers to learning among graduate students. Marks R, editor. Health Educ [Internet]. 2011 [cited 2018 Dec 31];111(5):425-45. Available from: https://doi.org/10.1108/09654281111161248.

7. Fleck MP, Louzada S, Xavier M, Chachamovich E, Vieira G, Santos L, et al. Aplicação da versão em português do instrumento abreviado de avaliação da qualidade de vida "WHOQOL-bref." Rev Saude Publica [Internet]. 2000 [cited 2018 Dec 31];34(2):178-83. Available from: http://dx.doi.org/10.1590/S0034-89102000000200012.

8. The Whoqol Group. The World Health Organization quality of life assessment (WHOQOL): Development and general psychometric properties. Soc Sci Med [Internet]. 1998 [cited 2018 Dec 31];46(12):1569-85. Available from:

https://doi.org/10.1016/S0277-9536(98)00009-4.

9. Moura IH, Nobre RS, Cortez RMA, Campelo V, Macêdo SF de, Silva ARV da. Qualidade de vida de estudantes de graduação em enfermagem. Rev Gaucha Enferm [Internet]. 2016;37(2). Available from: https://doi.org/10.1590/1983-1447.2016.02.55291. 10. Oliveira BM, Mininel VA, Felli VEA. Qualidade de vida de graduandos de enfermagem. Rev Bras Enferm [Internet]. 2011 [cited 2018 Dec 31];64(1):130-5. Available from: https://doi.org/10.1590/S0034-71672011000100019.

11. Bampi LNS, Baraldi S, Guilhem D, Pompeu RB, Campos ACO. Percepção sobre qualidade de vida de estudantes de graduação em enfermagem. Rev Gaucha Enferm [Internet]. 2013 [cited 2018 Dec 31];34(2):125-32. Available from:

https://doi.org/10.1590/S1983-14472013000200016.

12. Ciscon-Evangelista MR, Leal LDS, Oliveira NK, Menandro PRM. Pós-Graduação, formação profissional e postergação da constituição de família própria: um estudo com estudantes de mestrado e doutorado Psicologia e Saber Social [Internet]. 2013 [cited 2018 Dec 31];1(2):265-77. Available from: https://doi.org/10.12957/psi.saber.soc.2012.4908. 
13. Hoffman JC, Julie H. The academic transitional experiences of Masters' students at the University of the Western Cape. Curationis [Internet] 2012 [cited 2018 Dec 31];35(1):1-8. Available from: http://www.scielo.org.za/scielo.php?script=sci arttext\&pid=S2223-62792012000100010.

14. Santos AS, Perrone CM, Dias ACG. Adaptação à pós-graduação stricto sensu: uma revisão sistemática de literatura. Psico-USF [Internet]. 2015 [cited 2018 Dec 31];20(1):141-52. Available from: https://doi.org/10.1590/1413-82712015200113.

15. Mendes VR, lora JA. A opinião dos estudantes sobre as exigências da produção na pós-graduação. Rev. Bras. Ciênc. Esporte [Internet]. 2014 [cited 2018 Dec 31];36(1):171-87. Available from: https://doi.org/10.1590/S0101-32892014000100012.

16. Faro A. Estresse e estressores na pós-graduação: estudo com mestrandos e doutorandos no Brasil. Psic.: Teor. e Pesq. [Internet]. 2013 [cited 2018 Dec 31];29(1):51-60. Available from: https://doi.org/10.1590/S0102-37722013000100007. 17. Ministério da Educação, Coordenação de Aperfeiçoamento de Pessoal de Nível Superior. Plano Nacional de Pós-Graduação PNPG 2011-2020 [Internet]. Brasília: CAPES, 2010 [cited 2018 Dec 31]. v. 2. Available from:

https://www.capes.gov.br/images/stories/download/Livros-PNPG-Volume-I-Mont.pdf.

18. Perepiczka M, Balkin RS. Relationship Between Wellness and Age, Matriculation, and Relationship Status of Counselor Education Doctoral Students. J Humanist Couns Educ Dev [Internet]. 2010 [cited 2018 Dec 31];49(2):203-16. Available from: https://doi.org/10.1002/j.2161-1939.2010.tb00098.x.

19. Tzeng D, Chung W, Yang C. The Effect of Job Strain on Psychological Morbidity and Quality of Life in Military Hospital Nurses in Taiwan: A Follow-up Study. Ind Health [Internet]. 2013 [cited 2018 Dec 31];51(4):443-51. Available from: https://doi.org/10.2486/indhealth.2012-0013.

20. Lima EFA, Borges JV, Oliveira ERA, Velten APC, Primo CC, Leite FMC. Qualidade de vida no trabalho de enfermeiros de um hospital universitário. Rev. Eletr. Enf. [Internet]. 2013 [cited 2018 Dec 31];15(4):1000-6. Available from:

https://doi.org/10.5216/ree.v15i4.19546.

21. Dejours $C$, Abdoucheli E, Jayet C. Psicodinâmica do trabalho: contribuições da escola de dejouriana à análise da relação prazer, sofrimento e trabalho. São Paulo: Atlas, 2014. 152 p.

22. Galdino MJQ, Martins JT, Haddad MCFL, Ribeiro RP. Mestrado em enfermagem: sentimentos vivenciados por estudantes. Rev. enferm. UFPE on line. [Internet]. 2016 [cited 2018 Dec 31];10(2):501-7. Available from:

https://periodicos.ufpe.br/revistas/revistaenfermagem/article/view/10982.

23. Jamali A, Tofangchiha S, Jamali R, Nedjat S, Jan D, Narimani A, et al. Medical students' health-related quality of life: roles of social and behavioural factors. Med Educ [Internet]. 2013 [cited 2018 Dec 31];47(10):1001-12. Available from:

https://doi.org/10.1111/medu.12247.

24. Nehls N, Barber G, Rice E. Pathways to the PhD in Nursing: An Analysis of Similarities and Differences. J Prof Nurs [Internet]. 2016 [cited 2018 Dec 31];32(3):163-72. Available from: https://doi.org/10.1016/j.profnurs.2015.04.006.

25. Halse C, Bansel P. The learning alliance: ethics in doctoral supervision. Oxford Rev Educ [Internet]. 2012 [cited 2018 Dec 31];38(4):377-92. Available from: https://doi.org/10.1080/03054985.2012.706219. 